\title{
Exciting Hard Spheres
}

\author{
T. Antal, ${ }^{1}$ P. L. Krapivsky, ${ }^{2}$ and S. Redner ${ }^{2}$ \\ ${ }^{1}$ Program for Evolutionary Dynamics, Harvard University, Cambridge, MA 02138, USA \\ ${ }^{2}$ Center for Polymer Studies 83 Department of Physics, Boston University, Boston, MA 02215, USA
}

\begin{abstract}
We investigate the collision cascade that is generated by a single moving particle in a static and homogeneous hard-sphere gas. We argue that the number of moving particles at time $t$ grows as $t^{\xi}$ and the number collisions up to time $t$ grows as $t^{\eta}$, with $\xi=2 d /(d+2), \eta=2(d+1) /(d+2)$, and $d$ the spatial dimension. These growth laws are the same as those from a hydrodynamic theory for the shock wave emanating from an explosion. Our predictions are verified by molecular dynamics simulations in $d=1$ and 2. For a particle incident on a static gas in a half-space, the resulting backsplatter ultimately contains almost all the initial energy.
\end{abstract}

PACS numbers: 05.20.Dd: Kinetic theory, 45.50.Tn: Collisions, 47.40.Rs: Detonation waves

The classical hard-sphere gas serves as a paradigm for a real molecular gas for situations where quantum effects are negligible and where the intermolecular potential is also negligible except when two molecules are in close proximity. This model has been successfully applied to understand many statistical properties of equilibrium and non-equilibrium molecular fluids [1] .

The hard-sphere gas consists of identical spherical particles that move along straight-line constant-velocity trajectories that are interrupted by elastic collisions with other spheres. Despite its simplicity and considerable research devoted to this model, many unexpected properties continue to be discovered, such as the slow decay of velocity correlations [2] and the anomalous statistics of collision events [3]. Here we investigate a phenomenon of a somewhat different genre, namely, the response of a stationary hard-sphere gas to the perturbation of a single moving particle. After the first collision, two particles are moving and these, in turn, collide with other particles, ultimately leading to a remarkably symmetric cascade of moving particles (Fig 1). In this collision cascade, we argue that the number of moving particles at time $t$ grows as $t^{\xi}$ and the total number collisions up to time $t$ grows as $t^{\eta}$, with $\xi=2 d /(d+2), \eta=2(d+1) /(d+2)$, and $d$ is the spatial dimension.

The above setting is just the initial "break shot" in an infinite billiard table. In classical billiards theory, a wealth of beautiful phenomena has been discovered about periodic and chaotic motions of a single particle that collides elastically with a confining boundary [4, 5]. In real billiards, however, multiple particles move simultaneously. While some intriguing results exist about the collision dynamics of few-particle systems [ 6 ], little is known quantitatively about infinite-particle systems, such as the break shot that is the focus of this work.

The sudden injection of energy by the incident particle in the interior of a zero-temperature gas can also be regarded as an explosion, and the collision cascade is then analogous to the shock wave that propagates outward from the initial detonation. From hydrodynamic theory [7, 8], basic properties of the shock wave, such as its propagation velocity, and the radial dependences of the density and temperature profiles behind the wave are well understood. Strikingly, the collision cascade in the hard-sphere problem shares many quantitative properties of this shock wave.

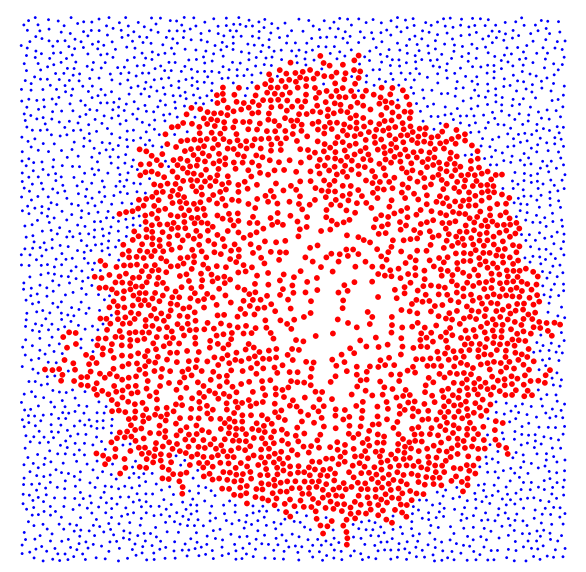

FIG. 1: (color online) Collision cascade in two dimensions at volume fraction $\rho=0.4$ and $t \approx 552$. Moving spheres are shown actual size (red) and stationary ones at half radius (blue). The cascade slowly moves rightward due to a particle initially at the center moving to the right.

Two natural characteristics of the cascade are $N_{t}$, the number of moving particles at time $t$, and $C_{t}$, the total number of collisions up to time $t$. These quantities depend on the initial sphere locations, but numerical simulations indicate that relative sample-to-sample fluctuations vanish in the long time limit. Therefore we focus on the number of moving particles and number of collisions averaged over many initial conditions, $N(t) \equiv\left\langle N_{t}\right\rangle$ and $C(t) \equiv\left\langle C_{t}\right\rangle$, respectively.

The number of moving particles can depend on time $t$, the density $n$, the spatial dimension $d$, the initial velocity $v_{0}$ of the moving particle, and the particle radii $a: N(t)=N\left[t, v_{0}, a, n, d\right]$. Since the particle number is dimensionless, dimensional analysis tells us that $N(t)$ should depend on the three independent dimensionless 
parameters $T=n^{1 / d} v_{0} t, \rho=n \Omega_{d} a^{d}$ and $d$ :

$$
N=N[T, \rho, d],
$$

where $\Omega_{d}=\pi^{d / 2} / \Gamma(1+d / 2)$ is the volume of unit sphere in $d$ dimensions. The self-similar nature of the collision cascade then suggests that $N(t)$ should have the powerlaw time dependence

$$
N \sim T^{\xi}
$$

with exponent $\xi$ that potentially depends on $\rho$ and $d$

We determine $\xi$ by writing a rate equation for the evolution of the number of moving particles. From Fig. 1) only particles within a shell of thickness one mean-free path $\ell \sim v \tau \sim 1 /\left(n a^{d-1}\right)$ from the edge of the cascade at time $t$ can recruit additional moving particles in a collision time $\tau$. We now assume equipartition in which the initial energy is shared approximately equally among the moving particles; simulations clearly support this assertion. At time $t$, each particle in the cascade then has energy that is roughly $N^{-1}$ times the initial energy, and root-mean-square velocity $v(t) \sim N^{-1 / 2}$. Using the observation from Fig. 1 that the collision cascade is compact, the number of particles within the collision shell is $n \ell R^{d-1}$. Consequently, the rate equation is

$$
\frac{d N}{d t} \sim \frac{n \ell R^{d-1}}{\tau} \sim n v\left(\frac{N}{n}\right)^{(d-1) / d} .
$$

Now using $v \sim N^{-1 / 2}$ and solving the resulting differential equation yields (2), with

$$
\xi=\frac{2 d}{d+2},
$$

a universal exponent that does not depend on $\rho$. Notice also that $N$ itself does not depend on $\rho$. We might anticipate that $\rho$ determines the time at which asymptotic behavior sets in, with this crossover time becoming long for small $\rho$.

As mentioned at the outset, the properties of the collision cascade can also be determined by a hydrodynamically-based dimensional analysis. The perturbation caused by a single moving particle can be viewed as the instantaneous release of energy $E=\frac{1}{2} m v_{0}^{2}$. This point energy source is akin to detonating an explosion, and the latter problem has an elegant scaling solution [7, 8] as long as the pressure behind the shock wave greatly exceeds that in front. This condition is always satisfied in our case, as the exterior gas has zero pressure. In this infinite Mach number limit, the radius $R(t)$ of the shock wave can only depend [7] on the energy release $E$, the mass density $n m$, where $m$ is the particle mass, and the time $t$. Therefore the radius must scale as

$$
R(t) \sim\left(E t^{2} / n m\right)^{1 /(d+2)},
$$

as this is the only variable combination with units of length. The only feature not determined by this dimensional analysis is the prefactor in Eq. (5) which requires solving the hydrodynamic equations of motion 4 , 8]. Using the result for $R(t)$ in conjunction with $E=\frac{1}{2} m v_{0}^{2}$ and $N=n \Omega_{d} R^{d}$, we again recover (2) \& (4).

To obtain the number of collisions $C(t)$ up to time $t$, we note that in a collision between a moving and a stationary particle one additional particle moves [9], while in a collision between moving particles their number remains the same. Thus we have the trivial bound $C(t) \geq N(t)$, which is valid for all times if we count the impulse that causes the initial particle motion as a collision. We again anticipate that the total number of collisions scales algebraically with time $C \propto T^{\eta}$.

To determine $\eta$, we write the rate equation for $C$

$$
\frac{d C}{d t} \sim \frac{N}{\tau}
$$

that merely states that $N$ additional collisions occur per collision time. We now use the fact that $\tau \sim \ell / v$ is proportional to $N^{1 / 2} / \rho$. Together with Eq. (2) for $N$ and $T=n^{1 / d} v_{0} t$, we integrate the rate equation to find

$$
C \sim \rho^{(d-1) / d} T^{1+\xi / 2} .
$$

Therefore $\eta=1+\xi / 2=2(d+1) /(d+2)$.

Although the initial trigger of the cascade is directional, the bulk motion of the cascade is negligible for $d>2$. Since the total momentum in the cascade equals the initial momentum $m v_{0}=1$, when the cascade encompasses $N$ particles, its center-of-mass velocity is given by $N v_{\mathrm{cm}}=1$, or $v_{\mathrm{cm}} \sim N^{-1} \sim T^{-\xi}$. Thus the position of the center-of-mass of the cascade is:

$$
x_{\mathrm{cm}}(t)=\int_{0}^{t} v_{\mathrm{cm}}\left(t^{\prime}\right) d t^{\prime} \sim \begin{cases}t^{1 / 3} & d=1 \\ \ln t & d=2 \\ \text { const. } & d>2\end{cases}
$$

To test our predictions, we perform molecular dynamics simulations on a gas of perfectly elastic hard spheres. In two dimensions, the system is initialized by placing, one-by-one, a given number of spheres of the same mass uniformly at random within a square box until a volume fraction $\rho$ is reached. If overlap is created when a sphere is introduced, one of the overlapping spheres is repositioned to eliminate this overlap. Without loss of generality we set the radius and the mass of spheres, and also the initial speed of the initially-moving sphere that is in the middle of the box to 1 . The remaining spheres are initially at rest. We stopped the simulations before any moving sphere reaches the edge of the box. Our data for the time dependence of the average number of moving spheres and the number of collisions is in good agreement with theory in the long-time limit (Fig. 2). We also find that the exponents $\xi$ and $\eta$ are close to the theoretical values of 1 and $3 / 2$ respectively, and are independent of the volume fraction $\rho$ for $0.1<\rho<0.45$. It is worth emphasizing that moving particles have their directions quickly randomized for $d \geq 2$ so that the cascade becomes symmetrical about its center of mass in spite of the directional initial perturbation. 


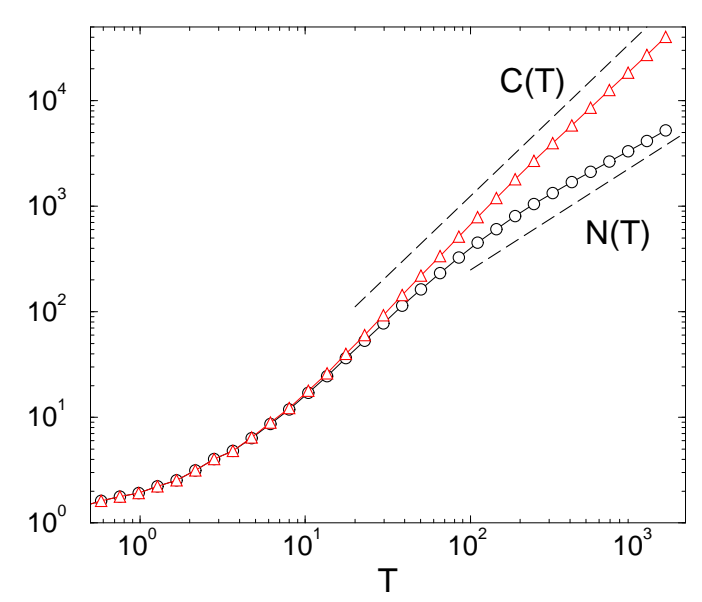

FIG. 2: Number of moving spheres $N(T)$ and number of collisions $C(T)$ versus scaled time $T=n^{1 / 2} v_{0} t$ in two dimensions for volume fraction 0.2 The dashed lines have slopes 1 and $3 / 2$ as predicted by theory.

The analogous problem in $d=1$ is pathological because there is no "mixing" — two elastically colliding particles merely switch their velocities. By this construction, the collision cascade reduces to the initial particle freely traversing the particle array. To avoid this pathology, we generalize slightly and allow for distributed particle masses. This generalization leads to collisional velocity mixing and thus represents a proper one-dimensional counterpart of our original system. We specifically consider uniformly distributed masses between 1 and $1-w$, with $w>0.2$ to avoid the slow approach to asymptotic behavior if the mass distribution is narrow. We initially place particles at integer points in the domain $x \in[-L, L]$; the particle at the origin is assigned a unit mass and velocity $v_{0}=1$.

With this initial condition, only particles in the region $x>0$ are excited at early times. However, the cascade gradually becomes more symmetrical! After an initial transient, the ratio of the positions of the rightmost and leftmost moving particles $\left|x_{+} / x_{-}\right|$systematically decreases and approaches a value that is close to 1 as $t \rightarrow \infty$. This same approach to symmetry occurs in the density profile of the gas. The collision cascade also satisfies the Rankine-Hugoniot conditions [8] that relate discontinuities in thermodynamic properties of the gas across the shock front in the infinite Mach number limit. For example, the gas just behind the leading edge of the cascade is twice as dense as that of the unperturbed gas (for a monoatomic gas in one dimension; the density ratio is $d+1$ in $d$ dimensions), in agreement with RankineHugoniot. Similar results have been found in large-scale numerical simulations of a planar shock in a hard-sphere fluid [10]. Finally, as shown in Fig. 3, our simulation results for $N(t)$ and $C(t)$ again agree with the theoretical predictions of $\xi=2 / 3$ and $\eta=4 / 3$ in $d=1$.

We now study the "splatter" when a particle is normally incident on a stationary gas in the half space $x \geq 0$

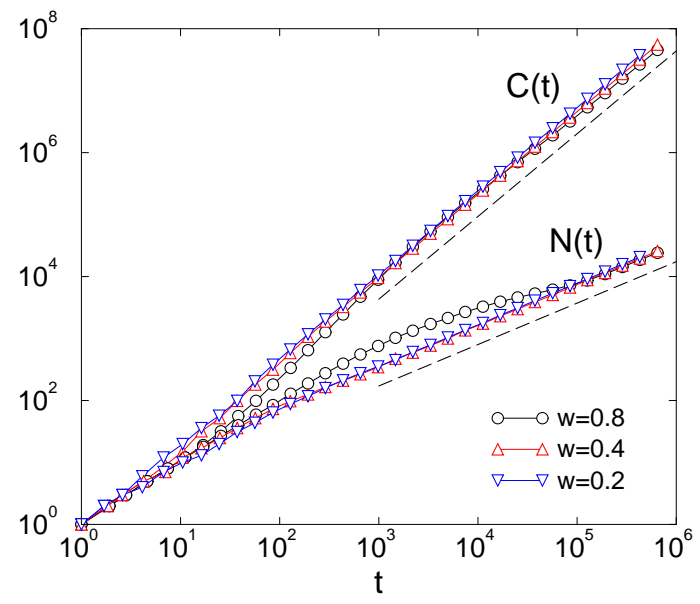

FIG. 3: (color online) Dependence of $N(T)$ and $C(T)$ versus scaled time $T=n v_{0} t$ in one dimension on a double logarithmic scale for different widths of the distribution of particle masses. The asymptotic behavior is approached more slowly for narrower mass distributions. The dashed lines have slopes $4 / 3$ and $2 / 3$ as predicted by theory.

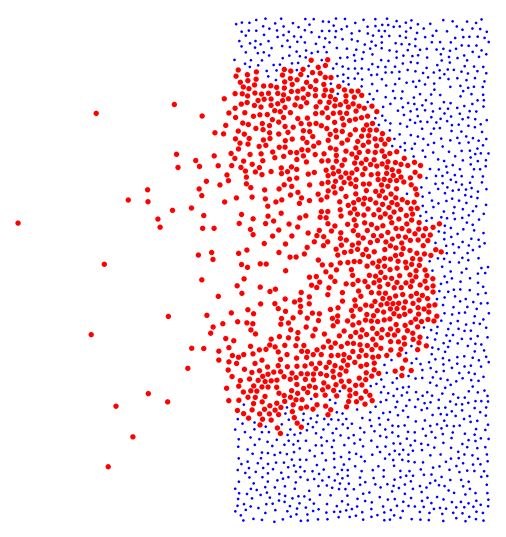

FIG. 4: (color online) Collision cascade in a two-dimensional half space at volume fraction $\rho=0.4$ and $t=400$ when a particle impacts from the left at $t=0$.

(Fig. 4). Again a collision cascade penetrates the gas in a manner similar to that of the bulk system, but some particles are ultimately ejected backward. Even though this backsplatter is visually a small fraction of the particles in the cascade, the energy contained in this backsplatter approaches value that is close to (and it perhaps equal to) the initial energy. In one dimension the situation is more clear but still surprising. At short times the incident particle continues moving rightward as it recollides with the particle array. Eventually, however, the incident particle is reflected so that it never experiences another collision. Particles 2, 3, .. undergo subsequent and similar reflections so that a finite fraction of the particles are ejected backward as a non-interacting "fan". The kinetic 
energy in the region $x>0$ slowly decreases with time and seems to vanish as $t^{-\beta}$ with $\beta \approx 0.1$, an observation that can be justified heuristically [1]. Thus all the energy ultimately resides in the region $x<0$ and, in fact, is concentrated in the trailing edge of the backsplatter. (A related issue of optimal energy transmission through a finite array was considered in [12].) An important consequence of the vanishing of the energy in the region $x>0$, is that Eq. (5) now predicts that the radius of the collision cascade grows as $t^{(2-\beta) / 3}$ instead of $t^{2 / 3}$ that arises from strict energy equipartition.

To summarize, the initial break shot on an infinite billiard table with a finite density of stationary spheres creates an almost symmetrical collision cascade within which excited particles share energy nearly equally. Dimensional arguments determine the extent of the cascade as a function of time. The properties of the cascade are closely analogous to those for a continuum shock wave that emanates from an explosion in a gas. It will be worthwhile to understand the limits of applicability of the continuum hydrodynamic analogy, especially for one dimension. The collisional splash problem reveals unexpected features, the most prominent being that apparently all of the energy is asymptotically carried by the backsplatter. A major challenge would be to prove that the energy transmitted to the initially occupied halfspace does indeed decay to zero; if so, to then determine the decay law and whether the splatter is described within a hydrodynamic framework.

We are grateful for financial support from NIH grant R01GM078986 (TA) and Jeffrey Epstein for support of the Program for Evolutionary Dynamics at Harvard University, as well as NSF grants CHE0532969 (PLK) and DMR0535503 (SR).
[1] P. Resibois and M. De Leener, Classical Kinetic Theory of Fluids (New York: Wiley, 1977).

[2] B. J. Alder and T. E. Wainwright, Velocity Autocorrelations for Hard Spheres, Phys. Rev. Lett. 18, 988 (1967).

[3] P. Visco, F. van Wijland, and E. Trizac, Collisional statistics of the hard-sphere gas, Phys. Rev. E 77, 041117 (2008).

[4] S. Tabachnikov, Billiards (Société Mathématique de France; Amer. Math. Soc., Providence, R.I., 1995).

[5] E. Gutkin, "Billiard in Polygons: Survey of Recent Results", J. Stat. Phys. 81, 7-26 (1996).

[6] D. Burago, S. Ferleger, and A. Kononenko, "A Geometric Approach to Semi-Dispersing Billiards." In: Hard Ball Systems and the Lorentz Gas, edited by D. Szasz (Berlin: Springer, 2000) and references therein. T. J. Murphy and E. G. D. Cohen, "On the Sequences of Collisions Among Hard Spheres in Infinite Space" ibid.

[7] L. I. Sedov, Similarity and Dimensional Methods in Mechanics (New York: Academic Press, 1959); G. I. Barenblatt, Scaling, Self-Similarity, and Intermediate Asymp- totics (Cambridge: Cambridge University Press, 1996).

[8] L. D. Landau and E. M. Lifshitz, Fluid Mechanics (New York: Pergamon Press, 1987).

[9] In a head-on collision between a moving and an identical stationary particle, the number of moving particles would not change; similarly, if particles meet with equal an opposite momenta they both would stop and the number of moving particles would decrease. However, such events occur with probability zero.

[10] C. Cercignani, A. Frezzotti, and P. Grosfils, "The Structure of an Infinitely Strong Shock Wave", Phys. Fluids 11, 2757 (1999); S. Takata, K. Aoki, and C. Cercignani, "The Velocity Distribution Function in an Infinitely Strong Shock Wave", Phys. Fluids 12, 2116 (2000).

[11] T. Antal, P. L. Krapivsky, and S. Redner, unpublished.

[12] T. Poeschel and N. V. Brilliantov. "Extremal Collision Sequences of Particles on a Line: Optimal Transmission of Kinetic Energy", Phys. Rev. E, 63, 021505 (2001). 